\title{
Thermal properties and time-dependent flow behavior of a viscous fluid
}

\author{
M. Imran Khan ${ }^{1, *}$, T. Hayat ${ }^{2,3}$, M. Ijaz Khan ${ }^{2}$, T. Yasmeen ${ }^{4}$ \\ ${ }^{1}$ Heriot Watt University, Edinburgh EH14 4AS, United Kingdom \\ ${ }^{2}$ Department of Mathematics, Quaid-I-Azam University 45320 Islamabad 44000, Pakistan \\ ${ }^{3}$ Nonlinear Analysis and Applied Mathematics (NAAM) Research Group, Department of Mathematics, Faculty of \\ Science, King Abdulaziz University, P. O. Box 80257, Jeddah 21589, Saudi Arabia \\ ${ }^{4}$ University of Engineering \& Technology, Peshawar, Pakistan
}

Received June 17, 2017; Accepted February 7, 2018

\begin{abstract}
Our goal in this attempt is to model a nonlinear stretchable flow of a radiative viscous liquid with magnetohydrodynamics. Flow caused is due to a unsteady stretching surface with variable thickness. Consideration of thermal radiation effect characterizes the heat transfer process. Induced electric and magnetic fields are not accounted for. Appropriate transformations gave nonlinear systems. Modern methodology, i.e., HAM, is implemented for the computational process. Velocity and temperature are plotted for influential variables which are important in this problem. Moreover, surface drag force and heat transfer rate are computed and discussed. Velocity field is noted to decay the function of the larger Hartman number whereas opposite situation for temperature is examined via larger radiation parameter.
\end{abstract}

Keywords: Thermal radiation; time-dependent flow; viscous liquid; variable sheet thickness

\section{INTRODUCTION}

The consideration of heat transport in stretchable flows is influential due to its broad utilizations in chemical engineering. Numerous processes in chemical engineering comprising polymer extrusion and metallurgical processes involved paper production, glass-fiber and chilling of molten liquid being extended into a chilling system [1]. The rates of cooling/stretching in such processes affect the excellence of the ultimate product. Various investigations in this direction have been reported under distinct physical aspects. For instance a magnetohydrodynamic (MHD) stretchable flow of viscous material considering velocity slip characteristics is reported by Mukhopadhyay [2]. Hayat et al. [3] modeled convected Jeffrey liquid with Joule heating and dissipation effects. Analysis of nonlinear micropolar and generalized Burgers liquids towards stretched surface are developed by Waqas et al. [4, 5]. Very recently Hayat et al. [6] scrutinized a stretchable flow of magneto-third-grade liquid with chemical reaction.

Several researchers nowadays are involved in exploring heat transfer characteristics via consideration of thermal radiation. This aspect regularly arises in several environmental and scientific processes. Few applications of thermal radiation comprise material dyeing processes, hot wiring, space/solar technology, glass fiber production, transpiration process, heat exchangers, prevention of energy and high temperature plasmas. Olajuwon et al. [7] explored Hall current and thermal radiation aspects in MHD micropolar liquid flow. Numerical solutions for thermally radiating threedimensional (3D) stretchable flow of magneto viscous nanoliquid with convective heating condition are

* To whom all correspondence should be sent:

E-mail: mk42@hw.ac.uk constructed by Mahanthesh et al. [8]. Hayat et al. [9, 10] reported a radiation impact in stagnation point flow of thixotropic and viscous materials towards stretched surface utilizing homotopic and numerical approach.

A magnetohydrodynamic (MHD) phenomenon has distinct industrial implication in mathematics, physics, engineering and chemistry. It has also applications in biological transportation, mixing of samples, cooling of strips, drug delivery, etc. This mechanism is fruitful to control both flow and heat transport. Various investigations have been reported in this context. For instance Sandeep and Sulochana [11] developed dual solutions for unsteady micropolar material with mixed convection and magnetohydrodynamics. Analysis of a three-dimensional dissipative stretchable flow of magneto Eyring-Powell nanoliquid with radiation and convective heating effects is reported by Mahanthesh et al. [12]. Khan and Khan [13] and Hayat et al. [14, 15] examined MHD impact in flow of power-law and Carreau materials through different geometries. Hayat et al. [16] explored thermal radiation and heat generation/absorption characteristics in convected Eyring-Powell nanoliquid towards stretched cylinder.

Aforestated literature survey assures that a stretchable flow regarding Newtonian/non-Newtonian materials is reported mostly considering negligible thickness. To our knowledge stretchable flows with variable thickness are not addressed extensively. Only few analyses in this direction are reported (for details see [17-19]). Thus our goal here is to venture further towards this aspect by considering unsteady stretched surface with variable thickness. Here we adopted the Newtonian fluid model with magnetohydrodynamics (MHD) and thermal radiation. The nonlinear developed systems are computed analytically via homotopy theory [20-25]. Moreover, the results are scrutinized graphically for distinct sundry variables. 


\section{Imran Khan et al.: Thermal properties and time-dependent flow behavior of a viscous fluid}

\section{FORMULATION}

Here the magnetohydrodynamic (MHD) flow of a viscous fluid towards unsteady stretching sheet with variable thickness is accounted for. The flow is generated due to a nonlinear stretching phenomenon with velocity $u_{w}(x)\left(=\frac{u_{0}(x+b)^{n}}{1-\gamma t}\right)$. Liquid is supposed in compression. Viscous liquid is conducted electrically subject to magnetic field of the form $B(x)\left(=B_{0}(x+b)^{\frac{n-1}{2}}\right)$ imposed in $y$ - direction. The effect of thermal radiation is retained. Besides this, viscous dissipation impact in heat process is neglected. The resulting boundary layer expressions of viscous fluid for present flow situation are:

$\frac{\partial u}{\partial x}+\frac{\partial v}{\partial y}=0$

$u \frac{\partial u}{\partial x}+v \frac{\partial u}{\partial y}=v \frac{\partial^{2} u}{\partial y^{2}}-\frac{\sigma B^{2}(x)}{\rho} u$,

$u \frac{\partial T}{\partial x}+v \frac{\partial T}{\partial y}=\frac{k}{\rho c_{p}} \frac{\partial^{2} T}{\partial y^{2}}$

$+\frac{16 \sigma^{*}}{3 k^{*} \rho c_{p}} \frac{\partial}{\partial y}\left(T^{3} \frac{\partial T}{\partial y}\right)$

with the subjected conditions:

$$
\left.\begin{array}{c}
u=u_{w}(x)=\frac{u_{0}(x+b)^{n}}{1-\gamma t}, v=0, \\
T=T_{w} \text { at } y=\delta(x+b)^{\frac{1-n}{2}}, \\
u \rightarrow 0, T \rightarrow T_{\infty} \text { as } y \rightarrow \infty .
\end{array}\right\}
$$

Here $(u, v)$ are the velocity components in the $(x, y)$ directions, respectively, $v$ the kinematic viscosity, $\sigma$ the electrical conductivity, $k$ the thermal conductivity, $\rho$ the fluid density, $\sigma^{*}$ the Stefan-Boltzmann constant, $k^{*}$ the mean absorption coefficient, $c_{p}$ the specific heat, $n$ the velocity power index, $B(x)=B_{0}(x+b)^{\frac{n-1}{2}}$ the non-uniform magnetic field, $\left(T, T_{w}, T_{\infty}\right)$ the (fluid, surface, ambient fluid) temperatures, $\delta$ the small variable w.r.t surface adequately thin, $u_{0}$ the reference velocity and $(b, \gamma)$ the positive constants.

Utilizing the following transformations:

$$
\begin{aligned}
u= & \frac{u_{0}(x+b)^{n}}{1-\gamma t} F^{\prime}(\xi), \\
v & =-\sqrt{\frac{n+1}{2} \frac{v u_{0}(x+b)^{n-1}}{1-\gamma t}}\left(F(\xi)+\xi \frac{n-1}{n+1} F^{\prime}(\xi)\right), \\
\xi= & y \sqrt{\frac{n+1}{2} \frac{u_{0}}{v(1-\gamma t)}(x+b)^{n-1}}, \\
& \Theta(\xi)=\frac{T-T_{\infty}}{T_{w}-T_{\infty}},
\end{aligned}
$$

incompressibility condition is satisfied trivially and Eqs. (2)-(5) become:

$$
\begin{aligned}
& F^{\prime \prime \prime}+F F^{\prime \prime}-\frac{2}{n+1} S\left(F^{\prime}-\frac{\eta}{2} F^{\prime \prime}\right)-\frac{2}{n+1} H a^{2} F^{\prime}=0, \\
& \left(1+\frac{4}{3} R\right) \Theta^{\prime \prime}+\operatorname{Pr} F \Theta^{\prime}-\frac{2}{n+1} S\left(2 \Theta+\frac{\eta}{2} \Theta^{\prime}\right)=0, \\
& F^{\prime}(\alpha)=1, F(\alpha)=\alpha \frac{1-n}{1+n}, \Theta(\alpha)=1, \\
& F^{\prime}(\infty)=0, \Theta(\infty)=0 \text {. }
\end{aligned}
$$

Now we define $F(\xi)=f(\xi-\alpha)=f(\eta)$ and $\Theta(\xi)=\theta(\xi-\alpha)=\theta(\eta)$ then Eqs. (7-9) become:

$$
\begin{aligned}
& \begin{array}{l}
f^{\prime \prime \prime}+f f^{\prime \prime}-\frac{2 n}{n+1} f^{\prime 2} \\
-\frac{2}{n+1} S\left(f^{\prime}-(\xi+\alpha) f^{\prime \prime}\right)-\frac{2}{n+1} H a^{2} f^{\prime}=0,
\end{array} \\
& \begin{array}{l}
\left(1+\frac{4}{3} R\right) \theta^{\prime \prime}+\operatorname{Pr} f \theta^{\prime} \\
-\frac{2}{n+1} S\left(2 \theta+\frac{\xi+\alpha}{2} \theta^{\prime}\right)=0,
\end{array} \\
& \left.\begin{array}{l}
f^{\prime}(0)=1, f(0)=\alpha \frac{1-n}{1+n}=0, \\
f^{\prime}(\infty)=0, \theta(0)=1, \theta(\infty)=0,
\end{array}\right\}
\end{aligned}
$$

where $S\left(=\frac{\gamma}{u_{0}(x+b)^{m-1}}\right)$ represents the unsteadiness parameter, $H a\left(=\frac{\sigma B_{0}^{2}}{\rho u_{0}}\right)$ the Hartman number, $R\left(=\frac{4 \sigma^{*} T_{\infty}^{3}}{k k^{*}}\right)$ the thermal radiation parameter, $\operatorname{Pr}\left(=\frac{\mu c_{p}}{k}\right)$ the Prandtl number and $\alpha\left(\delta \sqrt{\frac{n+1}{2} \frac{u_{0}}{v}}\right)$ the wall thickness parameter.

The skin friction coefficient and local Nusselt number are:

$$
C_{f}=\frac{\tau_{w}}{\rho u_{w}^{2}}, N u_{x}=\frac{(x+b) q_{w}}{k\left(T_{w}-T_{\infty}\right)},
$$

in which surface shear stress $\left(\tau_{w}\right)$ and surface heat flux $\left(q_{w}\right)$ are:

$$
\begin{aligned}
& \tau_{w}=\left.\mu \frac{\partial u}{\partial y}\right|_{y=\delta(x+b)^{\frac{1-n}{2}}}, \\
& q_{w}=\left.\left(-k\left(\frac{\partial T}{\partial y}\right)-\frac{16 \sigma^{*}}{3 k^{*} \rho c_{p}} \frac{\partial T}{\partial y}\right)\right|_{y=\delta(x+b)^{\frac{1\llcorner n}{2}}} .
\end{aligned}
$$

Utilizing Eq. (13) in Eq. (12) the skin friction coefficient and local Nusselt number in dimensionless forms are:

$$
\left.\begin{array}{l}
\sqrt{\operatorname{Re}_{x}} C_{f}=\sqrt{\frac{n+1}{2}} f^{\prime \prime}(0), \\
N u_{x} \operatorname{Re}_{x}^{-1 / 2}=-\left(1+\frac{4}{3} R\right) \sqrt{\frac{n+1}{2}} \theta^{\prime}(0),
\end{array}\right\}
$$

with $\operatorname{Re}_{x}=\frac{u_{w}(x)}{v}(x+b)$ as the local Reynolds number. 


\section{Imran Khan et al.: Thermal properties and time-dependent flow behavior of a viscous fluid SOLUTION METHODOLOGY}

Here we employed the HAM technique in order to acquire the convergent solutions of expressions (9) and (10) subject to the conditions given in Eq. (11). The relevant initial guesses and operators have the following forms:

$$
\begin{aligned}
& \left.\begin{array}{l}
f_{0}(\eta)=\alpha \frac{1-n}{1+n}+\left(1-e^{-\eta}\right), \\
\theta_{0}(\eta)=e^{-\eta},
\end{array}\right\} \\
& \left.\begin{array}{l}
£_{f}=f^{\prime \prime \prime}-f^{\prime}, £_{\theta}=\theta^{\prime \prime}-\theta, \\
\text { with } \\
£_{f}\left[C_{1}+C_{2} e^{\eta}+C_{3} e^{-\eta}\right]=0, \\
£_{\theta}\left[C_{4} e^{\eta}+C_{5} e^{-\eta}\right]=0,
\end{array}\right\}
\end{aligned}
$$

in which $C_{i}(i=1-5)$ elucidate the arbitrary constants. These constants are given as:

$$
\left.\begin{array}{l}
C_{2}=C_{4}=0, C_{3}=\left.\frac{\partial f_{m}^{*}(\eta)}{\partial \xi}\right|_{\eta=0}, \\
C_{1}=-C_{3}-f_{m}^{*}(0), C_{5}=-\theta_{m}^{*}(0) .
\end{array}\right\}
$$

\section{CONVERGENCE ANALYSIS}

The homotopic technique offers extraordinary choice to choose the auxiliary variables $\mathrm{h}_{f}$ and $\mathrm{h}_{\theta}$ concerning control and adjustment about the convergence. Now to determine the suitable values of $\mathrm{h}_{f}$ and $\mathrm{h}_{\theta}$ the $\hbar$-curves have been portrayed in Fig. 1. It is observed that allowable values are $-1.85 \leq \mathrm{h}_{f} \leq-0.15$ and $-1.6 \leq \mathrm{h}_{\theta} \leq$ 0.4 . Moreover, convergence of derived solutions is also ensured numerically through Table 1 . Clearly, $15^{\text {th }}$ order approximations are sufficient for the convergence of momentum and energy expressions.

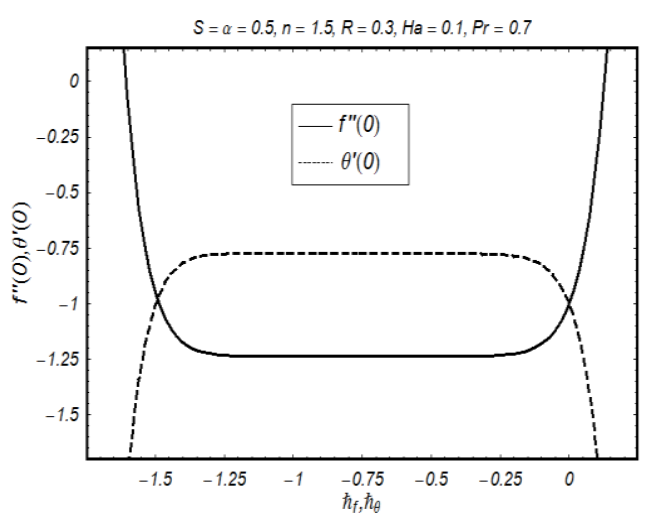

Fig. 1. h-curves for $\boldsymbol{f}^{\prime \prime}(\mathbf{0})$ and $\boldsymbol{\theta}^{\prime}(\mathbf{0})$.

Table 1. Homotopy solutions convergence when $\mathrm{S}=$ $\alpha=0.5, n=1.5, H a=0.1, R=0.3$ and $\operatorname{Pr}=0.7$.

\begin{tabular}{ccc}
\hline $\begin{array}{c}\text { Order of } \\
\text { approximations }\end{array}$ & $-f^{\prime \prime}(0)$ & $-\theta^{\prime}(0)$ \\
\hline 1 & 1.1895 & 0.8122 \\
\hline 5 & 1.2358 & 0.7745 \\
\hline 10 & 1.2359 & 0.7745 \\
\hline 15 & 1.2359 & 0.7745 \\
\hline 20 & 1.2359 & 0.7745 \\
\hline 25 & 1.2359 & 0.7745 \\
\hline
\end{tabular}

This section interprets the effects of influential variables like unsteadiness parameter $(S)$ shape parameter $(\alpha)$ velocity power index $(n)$ Hartman number (Ha) Prandtl number (Pr) and thermal radiation parameter $(R)$ on velocity $\left(f^{\prime}\right)$ temperature $(\theta)$ skin friction $\left(C_{f} \operatorname{Re}_{x}^{1 / 2}\right)$ and local Nusselt number $\left(N u \operatorname{Re}_{x}^{-1 / 2}\right)$. Figs. 2-9 and Tables 2 and 3 are presented for this purpose.

Impact of $S$ on $f^{\prime}$ is indicated in Fig. 2. Here larger $S$ shows smaller rate of stretching in the $x$-direction which ultimately reduce the velocity and thickness of boundary layer. Fig. 3 reveals the characteristics of $\alpha$ on $f$ ' Clearly $f$ ' and related thickness layer are reduced via larger $\alpha$. Physically when $\alpha$ is enhanced for $n<1$, stretching velocity decays which corresponds to a reduction of $f$ ' and related layer thickness. The role of $n$ on $f^{\prime}$ is reported in Fig. 4. Clearly, $f$ ' illustrates growing behavior via larger $n$. Higher $n$ corresponds to an enhancement in stretching rate which yields more deformation in the liquid. Thus velocity distribution boosts. The impact of $\mathrm{Ha}$ on $f^{\prime}$ is disclosed through Fig. 5. Clearly increase in $H a$ decays the velocity ( $\left.f^{\prime}\right)$ Since Lorentz force behaves as retarding force which increments the frictional resistance differing the liquid motion in the momentum layer thickness. Fig. 6 illustrates the effect of $\operatorname{Pr}$ on temperature $(\theta)$ Larger $\operatorname{Pr}$ considerably reduces temperature $(\theta)$ and the associated thermal boundary layer. As thermal diffusivity decays through larger Pr thus heat diffuses away slowly from the heated surface. Characteristics of radiation parameter on temperature $(\theta)$ are interpreted in Fig. 7. An increment in $R$ augments the heat flux from the sheet which corresponds to higher fluid temperature. Hence, the temperature $(\theta)$ and associated thermal layer boost with an increase in $R$. Fig. 8 describes the behavior of $S$ on temperature $(\theta)$ Clearly, temperature $(\theta)$ and associated layer thickness are decaying functions of $S$. Characteristics of $n$ on temperature $(\theta)$ are depicted in Fig. 9. As expected, both temperature and related thickness layer are enhanced via larger $n$.

The role of $S, \alpha$ and $H a$ on surface drag force is computed in Table 2. Here surface drag force enhances via larger $S$ and $H a$ while it decays when $\alpha$ is increased. Table 3 exhibits the impacts of $S, \operatorname{Pr}, R$ and $\alpha$ on Nusselt number. Clearly, Nusselt number augments via larger $S$; for $\operatorname{Pr}$ and $R$, however, opposite situation is noted when $\alpha$ is enhanced. 


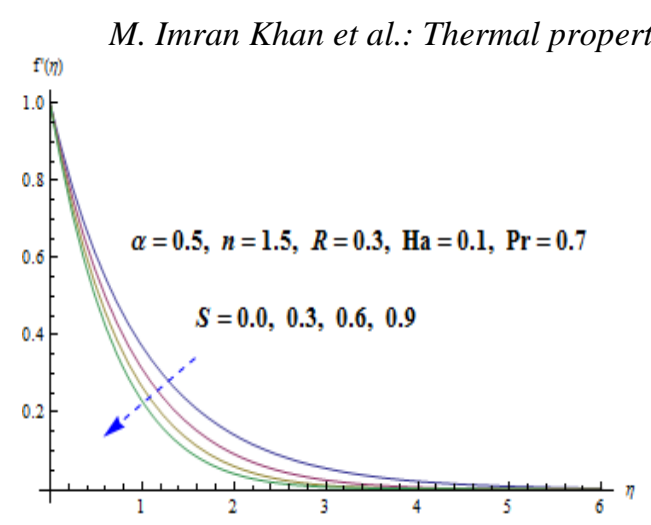

Fig. 2. $\boldsymbol{f}^{\prime}$ against $\mathrm{S}$.

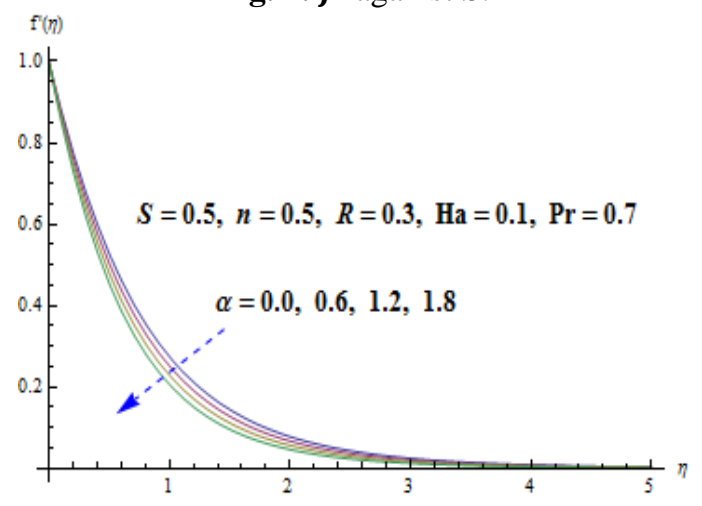

Fig. 3. $\boldsymbol{f}^{\prime}$ against $\alpha$.

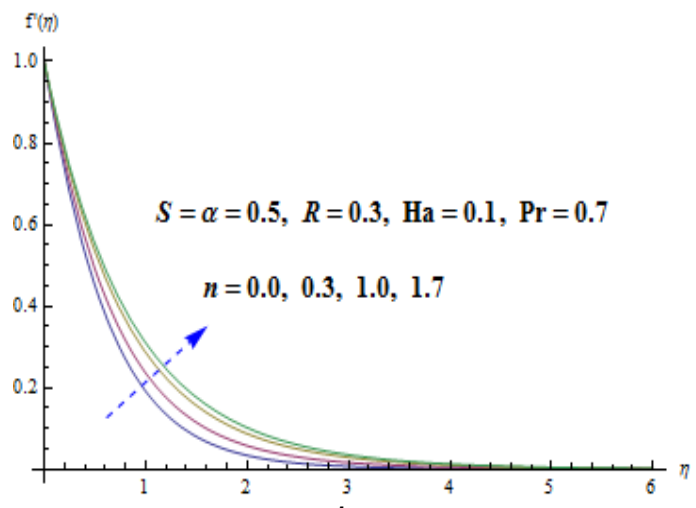

Fig. 4. $\boldsymbol{f}^{\prime}$ against $\mathrm{n}$.

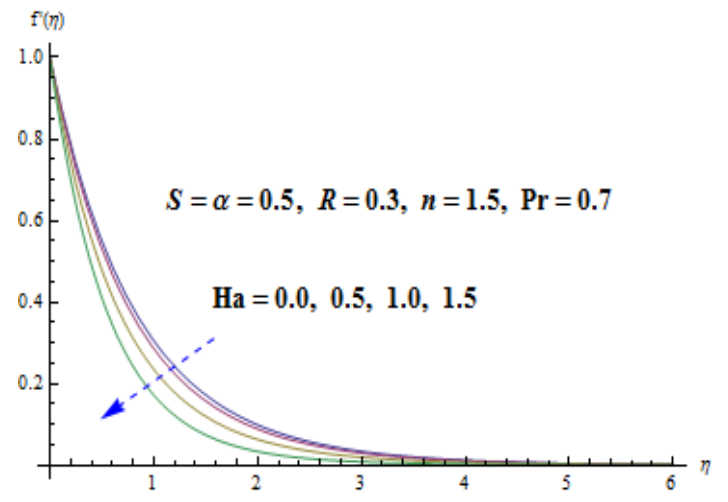

Fig. 5. $\boldsymbol{f}^{\prime}$ against Ha.

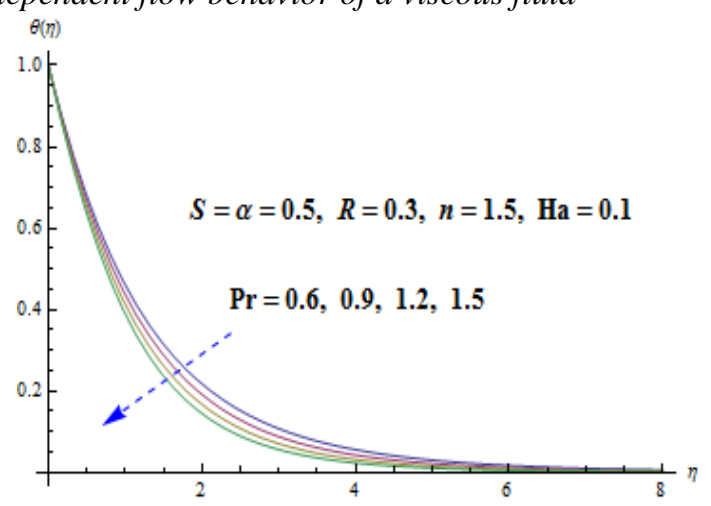

Fig. 6. $\boldsymbol{\theta}$ against $\operatorname{Pr}$.

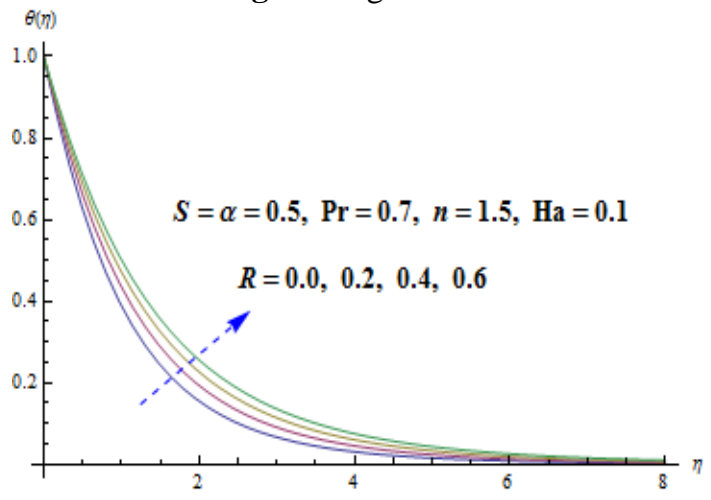

Fig. 7. $\boldsymbol{\theta}$ against R.

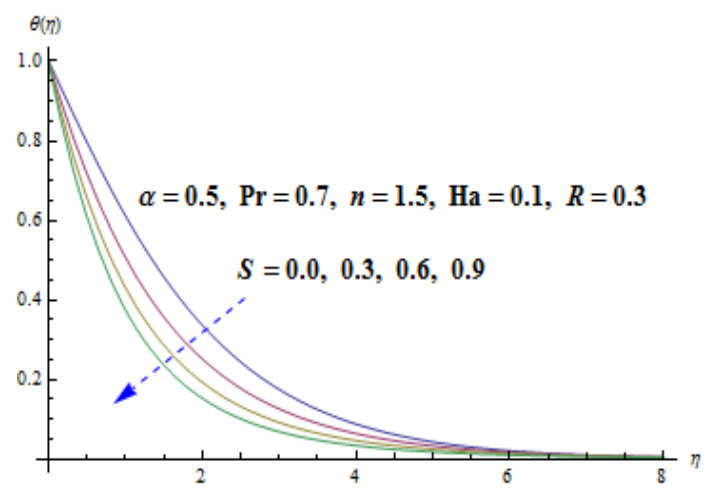

Fig. 8. $\boldsymbol{\theta}$ against $S$.

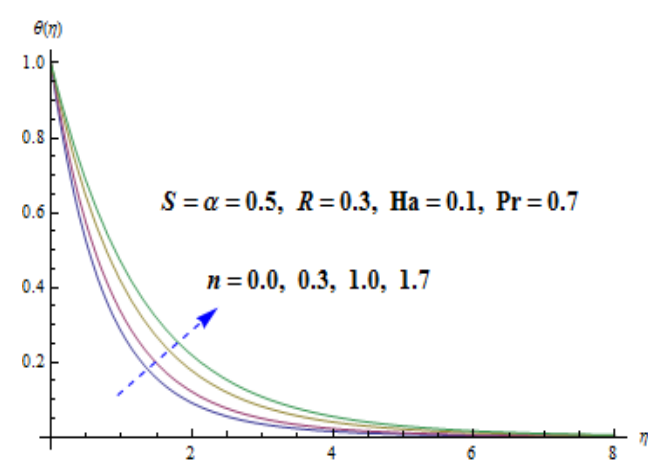

Fig. 9. $\boldsymbol{\theta}$ against $n$. 
M. Imran Khan et al.: Thermal properties and time-dependent flow behavior of a viscous fluid

Table 2. Effects of $S, \alpha$ and $H a$ on skin friction $\left(C_{\mathrm{j}} R e_{\mathrm{x}}{ }^{-1 / 2}\right)$ when $n=1.5, R=0.3$ and $\operatorname{Pr}=0.7$.

\begin{tabular}{cccc}
\hline$S$ & $\alpha$ & $\mathrm{Ha}$ & $-\theta^{\prime}(0)$ \\
\hline 0.0 & & & 1.1377 \\
\hline 0.3 & & & 1.2899 \\
\hline 0.6 & & & 1.4255 \\
\hline 0.5 & 0.0 & & 1.4377 \\
\hline & 0.6 & & 1.3709 \\
\hline & 1.2 & & 1.3073 \\
\hline & 0.5 & 0.0 & 1.3784 \\
\hline & & 0.4 & 1.4327 \\
\hline & & 0.8 & 1.5852 \\
\hline
\end{tabular}

Table 3. Effects of $S, \operatorname{Pr}, R$ and $\alpha$ on Nusselt number $\left(N u_{\mathrm{x}} R e_{\mathrm{x}}^{-1 / 2}\right)$ when $\mathrm{Ha}=0.1$ and $n=1.5$.

\begin{tabular}{|c|c|c|c|c|}
\hline$S$ & $P r$ & $R$ & $\alpha$ & $N u_{x} R e_{x}^{-0.5}$ \\
\hline 0.0 & & & & 1.3081 \\
\hline 0.3 & & & & 1.3981 \\
\hline 0.6 & & & & 1.4825 \\
\hline \multirow[t]{9}{*}{0.5} & 0.8 & & & 1.2265 \\
\hline & 0.9 & & & 1.2416 \\
\hline & 1.0 & & & 1.2569 \\
\hline & 0.7 & 0.0 & & 1.0271 \\
\hline & & 0.2 & & 1.1536 \\
\hline & & 0.4 & & 1.2666 \\
\hline & & 0.3 & 0.0 & 1.2496 \\
\hline & & & 0.6 & 1.2041 \\
\hline & & & 1.2 & 1.1602 \\
\hline
\end{tabular}

\section{CONCLUSIONS}

This research aims to explore the impact of unsteady variably thick surface in a magnetohydrodynamic (MHD) radiative flow of a viscous liquid. The presented research has the following key points:

- Unsteadiness parameter (S) has similar impact on velocity and temperature.

- Larger wall thickness parameter $(\alpha)$ and Hartman number $(\mathrm{Ha})$ reduce both the velocity and the related thickness of boundary layer.

- Temperature of the liquid improves via larger radiation factor $(R)$.

- Impacts of unsteadiness parameter $(S)$ and Prandtl number (Pr) on temperature distribution are similar in a qualitative manner.

- Surface drag force is noted to have a decaying function on larger wall thickness parameter $(\alpha)$, however, it boosts when unsteadiness parameter $(S)$ and Hartman number $(H \alpha)$ are enhanced.

- $\quad$ Impact of $S, \operatorname{Pr}$ and $R$ on Nusselt number is opposite to $\alpha$.

- The case for steady variably thick surface can be recovered by putting $\gamma=0$.

\section{REFREENCES}

1. H. Ajam, S. S. Jafari, N. Freidoonimehr, Ain Shams Eng. J., (2016), DOI: 10.1016/j.asej.2016.03.006
2. S. Mukhopadhyay, Ain Shams Eng. J., 4, 317 (2013).

3. T. Hayat, M. Waqas, S. A. Shehzad, A. Alsaedi, J. Hydrol. Hydromech., 63, 311 (2015).

4. M. Waqas, M. Farooq, M. I. Khan, A. Alsaedi, T. Hayat, T. Yasmeen, Int. J. Heat Mass Transf., 102, 766 (2016).

5. M. Waqas, T. Hayat, M. Farooq, S. A. Shehzad, A. Alsaedi, Cattaneo-Christov, J. Mol. Liq., 220, 642 (2016).

6. T. Hayat, M. I. Khan, M. Waqas, A. Alsaedi, T. Yasmeen, Chinese J. Chem. Eng., 25, (2017) 10.1016/j.cjche.2016.06.008.

7. B. I. Olajuwon, J. I. Oahimire, M. Ferdow, Eng. Sci. Tech. an Int. J., 17, 185 (2014).

8. B. Mahanthesh, B. J. Gireesha, R. S. R. Gorla, J. Nigerian Math. Soc., 35, 178 (2016).

9. T. Hayat, M. Waqas, S. A. Shehzad, A. Alsaedi, $J$. Mol. Liq., 215, 704 (2016).

10. T. Hayat, M. I. Khan, M. Waqas, A. Alsaedi, M. Farooq, Computer Methods Appl. Mech. Eng., 315, 1011 (2017).

11. N. Sandeep, C. Sulochana, Eng. Sci. Tech. an Int. J., 18, 738 (2015)

12. B. Mahanthesh, B. J. Gireesha, R. S. R. Gorla, J. Assoc. Arab Univ. Basic Appl. Sci., 23, (2017) DOI: 10.1016/j.jau as.2016.05.004.

13. M. Khan, W. A. Khan, AIP Adv., 6, 025211 (2016).

14. T. Hayat, S. Farooq, A. Alsaedi, B. Ahmad, Results Phys., 7, (2017). DOI: 10.1016/j.rinp.2016.12.048.

15. T. Hayat, M. I. Khan, M. Waqas, A. Alsaedi, Colloid Surf. A-Physicochem. Eng. Asp., 518, 263 (2017).

16. T. Hayat, M. I. Khan, M. Waqas, A. Alsaedi, J. Mol. Liq., 231, 126 (2017).

17. T. Fang, J. Zhang, Y. Zhong, Appl. Math. Comput., 218, 7214 (2012)

18. M. M. Khader, A. M. Megahed, European Physical Journal Plus, 100, 128 (2013).

19. M. I. Khan, T. Hayat, M. Waqas, A. Alsaedi, J. Mol. Liq., 230, 143 (2017).

20. S. J. Liao, Homotopic analysis method in nonlinear differential equations, Springer, Heidelberg, Germany, 2012.

21. T. Hayat, M. I. Khan, M. Farooq, T. Yasmeen, A. Alsaedi, J. Mol. Liq., 220, 1121 (2016).

22. T. Hayat, M. Waqas, M. I. Khan, A. Alsaedi, Int. J. Heat Mass Transf., 102, 1123 (2016).

23. M. Turkyilmazoglu, Filomat, 30, 1633 (2016).

24. T. Hayat, M. Waqas, S. A. Shehzad, A. Alsaedi, J. Mol. Liq., 215, 704 (2016).

25. T. Hayat, M. I. Khan, M. Farooq, A. Alsaedi, M. Waqas, T. Yasmeen, Impact of Cattaneo-Christov heat flux model in flow of variable thermal conductivity fluid over a variably thick surface, Int. J. Heat Mass Transfer, 99, 702 (2016).

26. T. Hayat, M. I. Khan, M. Waqas, A. Alsaedi, Results Phys., 7, 256 (2017). 